Gynecologic and

Obstetric Investigation
Gynecol Obstet Invest 2012;73:162-167

DOI: $\underline{10.1159 / 000332391}$
Received: April 13, 2011

Accepted after revision: August 18, 2011 Published online: January 31, 2012

\title{
The Correlation of Maternal Uric Acid Concentration with Small-for-Gestational-Age Fetuses in Normotensive Pregnant Women
}

\author{
Yoichiro Akahori Hisashi Masuyama Yuji Hiramatsu \\ Department of Obstetrics and Gynecology, Okayama University Graduate School of Medicine, Dentistry and \\ Pharmaceutical Sciences, Okayama, Japan
}

\section{Key Words}

Blood pressure $\cdot$ Creatinine $\cdot$ Small for gestational age •

Uric acid

\begin{abstract}
Aim: Elevated maternal serum uric acid is associated with small-for-gestational-age (SGA) fetuses. We investigated whether uric acid concentrations in normotensive pregnant women are correlated with fetal growth and related to kidney function. Methods: We carried out a case-control study using 40 patients who delivered SGA fetuses identified from the perinatal database and 80 patients who delivered appropriate-for-gestational-age (AGA) fetuses as the controls at Okayama University Hospital. Blood pressure, serum uric acid and creatinine level were measured in the patients' third trimester. Results: Maternal serum uric acid ( $p=0.0003)$ and creatinine $(p<0.0001)$ concentrations, as well as systolic and diastolic blood pressures ( $p=0.014$ and 0.037 , respectively), were significantly increased in the SGA group. There was a strong negative correlation between serum uric acid levels and birth weights $(r=-0.59 ; p=0.006)$ and a significant positive correlation between maternal serum uric acid and creatinine levels $(r=0.43 ; p<0.05)$ in cases of severe SGA $(<5$ th percentile). Multiple linear regression analysis indicated that
\end{abstract}

uric acid is an independent risk factor for SGA. Conclusions: Increasing maternal uric acid concentrations were associated with slightly impaired kidney function and SGA in normotensive pregnant women.

Copyright $\odot 2012$ S. Karger AG, Basel

\section{Introduction}

During pregnancy, circulating uric acid levels are regulated by alterations in renal handling. Maternal serum uric acid levels decrease by $25-35 \%$ during the first trimester due to an increase in glomerular filtration rate and a decrease in reabsorption in the proximal tubule. There is a subsequent rise to prepregnancy levels near term, which is related to a decrease in uric acid clearance due to postsecretory reabsorption [1-4].

Hyperuricemia in pregnancy is associated with adverse fetal outcome and preeclampsia. Uric acid directly inhibits amino acid transfer in the placenta and suppresses fetal growth [2]. Elevated levels of uric acid may have a proliferative and proinflammatory effect on the small blood vessels of the placenta, resulting in small-for-gestational-age (SGA) fetuses [5]. Uric acid stimulates the production of vasoconstrictors and inflammatory agents, re-

\section{KARGER \\ Fax +4161306 1234 \\ E-Mail karger@karger.ch}

www.karger.com
(C) 2012 S. Karger AG, Basel

$0378-7346 / 12 / 0732-0162 \$ 38.00 / 0$

Accessible online at:

www.karger.com/goi
Hisashi Masuyama, MD, PhD

2-5-1 Shikata

Okayama 700-8558 (Japan)

Tel. +81 862357320

E-Mail masuyama@cc.okayama-u.ac.jp 
duces nitric oxide production, and increases thromboxane generation in vascular smooth muscle cells [6-9]. Thus, hyperuricemia is strongly associated with endothelial cell dysfunction, and elevated serum uric acid levels usually precede hypertension.

In patients with preeclampsia, the association between maternal uric acid levels and birth weights has been investigated $[10,11]$. Furthermore, in normotensive pregnant women, uric acid concentrations in the second trimester correlated with insulin resistance and lower birth weights [12]. Presently, there are no reports describing the association between hyperuricemia and birth weight with respect to renal functions.

In this study, we related uric acid concentrations in the third trimester to birth weight among normotensive pregnant women. We evaluated the association between maternal serum uric acid levels and intrauterine fetal growth in relation to serum creatinine levels as a measure of maternal renal function.

\section{Subjects and Methods}

\section{Study Design}

We carried out a case-control study using 40 patients who delivered SGA fetuses (SGA group) identified from the perinatal database from 2005 to 2009 , and 80 patients who delivered appropriate-for-gestational-age (AGA) fetuses as the controls (AGA group) in 2009 at the Okayama University Hospital, who were consecutive subjects in each of the groups. Blood pressure was recorded as the average of three measurements. Diastolic blood pressure was determined by a mercury sphygmomanometer using the fifth Korotkoff sound after a 5 -min rest in sitting position when the patients visited our outpatient office during the morning. Pregnant women were normotensive, defined as a systolic blood pressure $<140 \mathrm{~mm} \mathrm{Hg}$ and a diastolic blood pressure $<90$ $\mathrm{mm} \mathrm{Hg}$. According to Japanese standards, SGA was defined as a birth weight $<10$ th percentile, severe SGA was $<5$ th percentile, and AGA was $>10$ th but $<90$ th percentile. Exclusion criteria were: SGA due to congenital abnormality, chromosomal abnormality, maternal complications including diabetes mellitus and collagen diseases, multiple birth, and umbilical cord factors; maternal use of alcohol and nicotine; and preterm birth. There were no pregnant women with either AGA or SGA who developed preeclampsia or hypertension during labor or after delivery.

Baseline demographic information collected from the database included: maternal height, weight before pregnancy, weight at the time of delivery, weight gain during pregnancy, body mass index (BMI) before pregnancy, maternal blood pressure, gestational age (weeks) at delivery, the newborn's gender, birth weight (in grams; percentile as a measure of the severity of SGA), Apgar score, and placental weight.

This study was approved by the Institutional Ethical Review Board of Okayama University Hospital (project No. 186, June 21st, 2004), and all subjects gave their informed consent.

\section{Laboratory Analyses}

Blood was collected in the third trimester before the start of labor. Serum samples were immediately prepared by centrifugal separation. Serum uric acid (Sino-test Co., Tokyo, Japan) and creatinine (Mizuho Medy Co., Ltd., Saga, Japan) levels were measured using colorimetric assay kits according to the manufacturer's instructions. The coefficients of variance were 6 and $8 \%$, respectively.

\section{Statistical Analysis}

Data are presented as means $\pm \mathrm{SD}$. All comparisons between the SGA and AGA groups were carried out using the Mann-Whitney $U$ test, except for gender and the proportion of primigravidas, which were analyzed with the $\chi^{2}$ test. Correlation between variables was conducted with Spearman's rank correlation. Multiple linear regression analysis was performed to determine which factors were significantly and independently correlated with severe SGA. The following parameters were included in the model: uric acid concentrations, placental weights, mean blood pressure, and weight gains during pregnancy. A p value of $<0.05$ was considered significant. Statistical analyses were conducted using JMP statistical discovery software (SAS Institute, Cary, N.C., USA).

\section{Results}

\section{Patient Characteristics}

There were no significant differences in maternal age, the proportion of primigravidas, height, weight and BMI before pregnancy, and weight gain during pregnancy, between the SGA and AGA groups. The mean birth weight (AGA: 2,913 \pm 282 g; SGA: 2,304 \pm 271 g; $\mathrm{p}<0.0001$ ) and placental weight (AGA: $570 \pm 95 \mathrm{~g}$; SGA: $420 \pm 58 \mathrm{~g}$; $\mathrm{p}<0.0001)$ were significantly lower in the SGA group, although the mean gestational age at delivery (AGA: 38.0 \pm 0.8 weeks; SGA: $38.6 \pm 1.33$ weeks; $p=0.02)$ was significantly higher. Mean systolic (AGA: $108.6 \pm 13.1 \mathrm{~mm}$ Hg; SGA: $115.2 \pm 10.8 \mathrm{~mm} \mathrm{Hg}$; $=0.014$ ) and diastolic blood pressures (AGA: $65.8 \pm 10.6 \mathrm{~mm} \mathrm{Hg}$; SGA: $70.1 \pm$ $9.6 \mathrm{~mm} \mathrm{Hg} ; \mathrm{p}=0.037$ ), and the mean blood pressure [i.e. diastolic pressure $+1 / 3$ (systolic pressure - diastolic pressure); AGA: $80.0 \pm 10.9 \mathrm{~mm} \mathrm{Hg}$; SGA: $85.1 \pm 9.5 \mathrm{~mm}$ $\mathrm{Hg} ; \mathrm{p}=0.016$ ] were significantly higher in the SGA group, although they remained within the normal range (table 1).

The incidence of cesarean section in pregnant women with AGA was high compared to those with SGA (93.8 vs. $55 \%$ ). Delivery by cesarean section allowed us to obtain clinical laboratory data including creatinine and uric acid levels, because normal pregnant women who vaginally deliver do not routinely receive blood examinations for renal and liver functions in our hospital. The method of selection for pregnant women with AGA might have af- 
Table 1. Patients' characteristics

\begin{tabular}{lccc}
\hline & AGA $(\mathrm{n}=80)$ & SGA $(\mathrm{n}=40)$ & p value \\
\hline Age, years & $31.4 \pm 4.76$ & $31.1 \pm 5.49$ & $\mathrm{~ns}$ \\
Primipara/multipara & $54 / 26$ & $27 / 13$ & $\mathrm{~ns}$ \\
Height, cm & $157.4 \pm 5.6$ & $158.0 \pm 4.19$ & $\mathrm{~ns}$ \\
Body weight (pre-pregnancy), kg & $52.4 \pm 9.0$ & $51.1 \pm 7.2$ & $\mathrm{~ns}$ \\
BMI & $21.1 \pm 3.5$ & $20.3 \pm 2.4$ & $\mathrm{~ns}$ \\
Body weight gain during pregnancy, kg & $8.9 \pm 3.8$ & $7.3 \pm 4.2$ & $\mathrm{~ns}$ \\
Systolic blood pressure, mm Hg & $108.6 \pm 13.1$ & $115.2 \pm 10.8$ & 0.014 \\
Diastolic blood pressure, mm Hg & $65.8 \pm 10.6$ & $70.0 \pm 9.6$ & 0.037 \\
Mean blood pressure, mm Hg & $80.0 \pm 10.9$ & $85.1 \pm 9.5$ & 0.016 \\
Gestational age at blood sampling, weeks & $35.9 \pm 1.4$ & $36.3 \pm 1.9$ & $\mathrm{~ns}$ \\
Placenta, g & $570 \pm 95$ & $420 \pm 58$ & $<0.0001$ \\
Gestational age at delivery, weeks & $38.0 \pm 0.8$ & $38.6 \pm 1.33$ & 0.02 \\
Gender, male/female & $40 / 40$ & $21 / 19$ & $\mathrm{~ns}$ \\
Birth weight, g & $2,913 \pm 282$ & $2,304 \pm 271$ & $<0.0001$ \\
Birth weight, percentile & $46.8 \pm 23.0$ & $4.5 \pm 2.2$ & $<0.0001$ \\
Height at birth, cm & $48.3 \pm 1.8$ & $46.8 \pm 2.2$ & 0.0005
\end{tabular}

Unless noted, values are the mean \pm SD. ns = Not significant. Statistical analysis was conducted by MannWhitney's U test except for 'Primipara/multipara' and 'Gender' which were conducted by $\chi^{2}$ test.

fected the average of gestational weeks at delivery. In the SGA group, 14 elective cesarean sections were planned due to previous myomectomy, previous Jones and Jones operation, breech presentation or previous cesarean section, and 8 emergent operations were performed due to nonreassuring fetal status. In the AGA group, 75 elective cesarean sections were planned due to breech presentation, previous myomectomy or previous cesarean section. However, there was no significant difference in gestational age at sampling and all samples in both groups were obtained before labor onset.

\section{Maternal Uric Acid Levels}

The mean gestational age at collection of serum samples was not significantly different between the two groups (AGA: $35.9 \pm 1.4$ weeks; SGA: $36.3 \pm 1.9$ weeks; table 1). The mean maternal serum uric acid level (SGA: $0.29 \pm 0.07 \mathrm{mmol} / \mathrm{l}$; AGA: $0.24 \pm 0.05 \mathrm{mmol} / \mathrm{l} ; \mathrm{p}=$ 0.0003 ; fig. 1a) and maternal serum creatinine level (SGA: $48.3 \pm 7.7 \mu \mathrm{mol} / \mathrm{l}$; AGA: $42.3 \pm 6.6 \mu \mathrm{mol} / \mathrm{l} ; \mathrm{p}<$ 0.0001 ; fig. $1 \mathrm{~b}$ ) were significantly higher in the SGA group than in the AGA group. However, their fluctuations were within the reference range for nonpregnant women. The mean uric acid level of SGA at umbilical cord blood $(0.30 \pm 0.07 \mathrm{mmol} / \mathrm{l}, \mathrm{n}=10)$ was significantly increased compared with AGA $(0.25 \pm 0.08 \mathrm{mmol} / \mathrm{l}$, $\mathrm{n}=21)(\mathrm{p}=0.005)$.

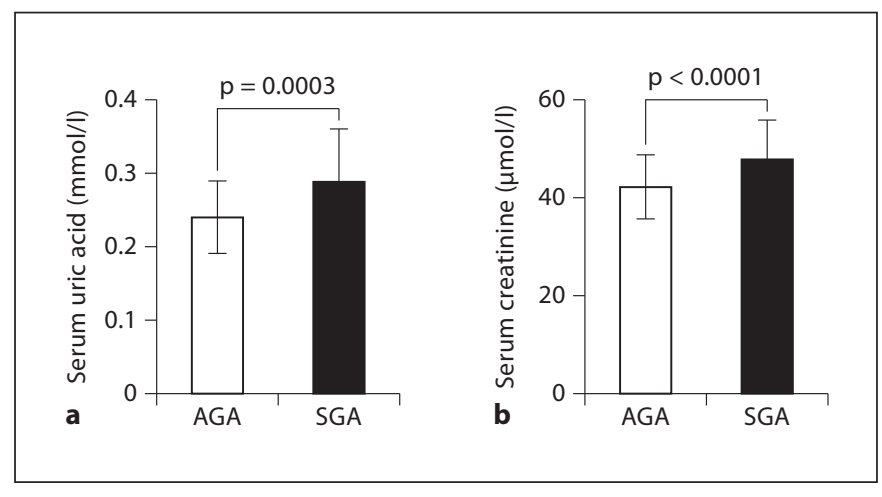

Fig. 1. Comparisons of maternal uric acid level (a) and creatinine level (b) between the SGA and the AGA group.

\section{Maternal Uric Acid Levels and Severe SGA}

Twenty cases of SGA were diagnosed as severe with a birth weight $<5$ th percentile and the mean maternal serum uric acid level and creatinine level were $0.31 \pm 0.08$ $\mathrm{mmol} / \mathrm{l}$ and $47.7 \pm 9.4 \mathrm{mmol} / \mathrm{l}$, respectively. In the severe SGA group, there was a significant negative correlation between birth weight percentile and maternal serum uric acid levels ( $\mathrm{r}=-0.59 ; \mathrm{p}=0.006$; fig. $2 \mathrm{a}$ ), and a significant positive correlation between maternal serum uric acid and creatinine levels $(r=0.43 ; p<0.05$; fig. $2 b)$. There 


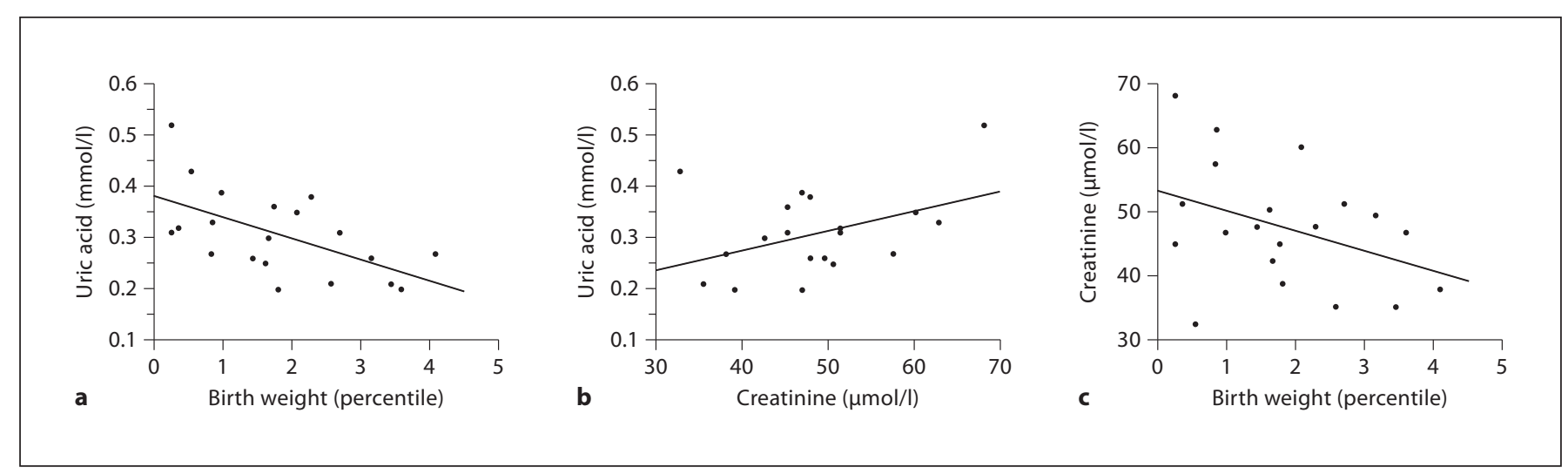

Fig. 2. Correlations of birth weight and maternal uric acid concentration (a), maternal uric acid and creatinine concentrations (b) and birth weight and maternal creatinine concentration (c) in the severe SGA group.

Table 2. Multiple linear regression analysis of factors associated with degree of severe SGA

\begin{tabular}{lll}
\hline Factor & $\beta$ & $\mathrm{p}$ value \\
\hline Uric acid, mmol/l & -0.51 & 0.004 \\
Placenta, g & 0.001 & 0.65 \\
Mean blood pressure, mm Hg & 0.003 & 0.90 \\
Weight gain, kg & 0.11 & 0.03 \\
$\mathrm{R}^{2}$ & 0.55 & 0.01 \\
\hline
\end{tabular}

were no significant correlations between birth weight percentile and maternal serum creatinine levels $(\mathrm{r}=$ -0.39 ; fig. 2c) and between birth weight percentile and the systolic, diastolic, and mean blood pressures (data not shown). There were no significant correlations among each factor examined here in the mild SGA group and in the control group. By forward selection method, uric acid, placental weight, mean blood pressure and weight gain were chosen as the independent factors, but creatinine was not chosen. Multiple linear regression analysis using maternal serum uric acid levels, maternal mean blood pressures, placental weights, and maternal weight gains during pregnancy as continuous variables, and birth weight percentile as the objective variable (table 2) indicated that maternal uric acid levels and maternal weight gain during pregnancy were associated with severe SGA. We also analyzed systolic and diastolic blood pressure as continuous variables, but they were not associated with severe SGA.

Uric Acid and Fetal Growth in Normotensive Pregnancy

\section{Discussion}

In this study, we evaluated the relationship between maternal serum uric acid levels and birth weights in normotensive pregnant women. We found elevated maternal uric acid levels in the third trimester in women diagnosed with SGA, but a significant negative correlation between maternal uric acid levels and birth weight percentiles in cases of severe SGA. The associations between hyperuricemia with preeclampsia and SGA $[10,11]$ and hyperuricemia with insulin resistance and lower birth weight in normotensive women in midpregnancy have previously been reported [12]. These data suggest that elevated uric acid concentrations might have a direct or indirect effect on fetal growth.

Elevated uric acid levels were negatively correlated with fetal growth. However, the variations in uric acid levels were within the reference range measured in healthy human serum $(0.12-0.39 \mathrm{mmol} / \mathrm{l})$. Hyperuricemia is associated with components of metabolic syndrome. The difference in serum uric acid levels between patients with metabolic syndrome and controls can be as low as $0.03-$ $0.06 \mathrm{mmol} / \mathrm{l}[13-15]$, and the average serum uric acid concentration of patients with metabolic syndrome is 0.35 $\mathrm{mmol} / \mathrm{l}[15]$. These data indicate that the fluctuations in serum uric acid levels between the SGA $(0.29 \mathrm{mmol} / \mathrm{l})$ and the AGA $(0.24 \mathrm{mmol} / \mathrm{l})$ group in our study are similar to the difference between metabolic syndrome and normal controls, suggesting that this variation might be significant for fetal growth.

We used serum creatinine concentrations to evaluate maternal renal function. Information describing renal

Gynecol Obstet Invest 2012;73:162-167 
functions during normal pregnancy is limited. Renal function in nonpregnant women is assessed using a predictive equation developed from the Modification of Diet in Renal Disease study, which calculates an estimated glomerular filtration rate [16]. However, this calculation underestimates the GFR in healthy pregnant women and those with preeclampsia [17]. In these cases, maternal serum creatinine levels and creatinine clearance may provide a more accurate assessment of renal function [17]. We found a slight increase in maternal serum creatinine in the SGA group, which implied that renal function was deteriorating. As maternal serum creatinine and uric acid levels showed a positive correlation, inferior maternal renal function in the SGA group may have caused the increase in serum uric acid concentrations, or the clearances of uric acid and creatinine from the circulation may be affected by similar mechanisms at the same time in the SGA group. Because we have no additional data about the relationship between uric acid and other markers for renal function, further analysis is required to investigate the relationship between serum uric acid levels and renal function during pregnancy.

In SGA patients, maternal blood pressure was significantly elevated, probably in association with the higher uric acid levels. Hyperuricemia is known to induce vascular smooth muscle cell proliferation and inflammation, resulting in increased blood pressure $[5,7-9]$. The fluctuations in maternal blood pressure were all within the normal range, therefore the clinical implications of our findings are unknown. Increased levels of serum uric acid may damage the placental vasculature and result in preeclampsia [6]. Furthermore, elevated serum uric acid in pregnant women is associated with SGA, possibly due to a reduction in placental uptake of amino acids [18]. The higher maternal uric acid levels in our SGA patients may be involved in the mechanisms resulting in reduced fetal growth.
Uric acid is the end product of purine metabolism, and it is primarily excreted through the kidneys. In serum, the concentration of uric acid in healthy individuals is maintained at $0.35 \mathrm{mmol} / 1$ or less; hyperuricemia is defined as a serum uric acid concentration of $>0.35 \mathrm{mmol} / 1$ [19]. When hyperuricemia develops, cardiovascular disease, renal failure, gout and urinary stones, metabolic syndrome, and insulin resistance can occur [20, 21]. Furthermore, uric acid passes easily though the placenta by simple diffusion [22]. We observed that the mean concentration of uric acid in placental blood were significantly different between the two groups. According to the Barker hypothesis, in utero exposure to hyperuricemia might alter developmental programming resulting in a decreased number of nephrons and a predisposition to insulin resistance $[12,23,24]$. In future, this interesting aspect should be further investigated.

In this study, SGA occurred in women with slightly elevated blood pressure and lower renal functions that were within the normal nonpregnant range; the characteristics of the group were not consistent with the diagnostic criteria for preeclampsia. We conclude that slightly impaired kidney function might result in increased maternal uric acid, which was associated with SGA in normotensive women. Our data also suggested that uric acid level might a potential marker for slightly impaired kidney function and SGA in normotensive pregnant women for daily clinical obstetric management.

\section{Acknowledgement}

This work was supported in part by research grants (17591739) from the Ministry of Education, Science and Culture of Japan.

\section{References}

1 Boyle JA, Campbell S, Duncan AM, Greig WR, Buchanan WW: Serum uric acid levels in normal pregnancy with observations on the renal excretion of urate in pregnancy. J Clin Pathol 1966;19:501-513.

$\checkmark 2$ Dunlop W, Davison JM: The effect of normal pregnancy upon the renal handling of uric acid. Br J Obstet Gynaecol 1977;84:13-21.

$\checkmark 3$ Lind T, Godfrey KA, Otun H, Philips PR: Changes in serum uric acid concentrations during normal pregnancy. Br J Obstet Gynaecol 1984;91:128-132.

4 Semple PF, Carswell W, Boyle JA: Serial studies of the renal clearance of urate and inulin during pregnancy and after the puerperium in normal women. Clin Sci Mol Med 1974;47: $559-565$.

5 Watanabe S, Kang DH, Feng L, Nakagawa T, Kanellis J, Lan H, Mazzali M, Johnson RJ: Uric acid, hominoid evolution, and the pathogenesis of salt-sensitivity. Hypertension 2002;40:355-360.

6 Bainbridge SA, Roberts JM: Uric acid as a pathogenic factor in preeclampsia. Placenta 2008;29Suppl A:S67-S72.

7 Kang DH, Nakagawa T, Feng L, Watanabe S, Han L, Mazzali M, Truong L, Harris R, Johnson RJ: A role for uric acid in the progression of renal disease. J Am Soc Nephrol 2002;13: 2888-2897. 
8 Kang DH, Park SK, Lee IK, Johnson RJ: Uric acid-induced C-reactive protein expression: implication on cell proliferation and nitric oxide production of human vascular cells. J Am Soc Nephrol 2005; 16:3553-3562.

-9 Mazzali M, Kanellis J, Han L, Feng L, Xia YY, Chen Q, Kang DH, Gordon KL, Watanabe S, Nakagawa T, Lan HY, Johnson RJ: Hyperuricemia induces a primary renal arteriolopathy in rats by a blood pressure-independent mechanism. Am J Physiol Renal Physiol 2002;282:F991-F997.

$\checkmark 10$ Redman CW, Beilin LJ, Bonnar J, Wilkinson $\mathrm{RH}$ : Plasma-urate measurements in predicting fetal death in hypertensive pregnancy. Lancet 1976;1:1370-1373.

-11 Roberts JM, Bodnar LM, Lain KY, Hubel CA, Markovic N, Ness RB, Powers RW: Uric acid is as important as proteinuria in identifying fetal risk in women with gestational hypertension. Hypertension 2005;46:12631269.

12 Laughon SK, Catov J, Roberts JM: Uric acid concentrations are associated with insulin resistance and birthweight in normotensive pregnant women. Am J Obstet Gynecol 2009;201:582.e1-e6.
13 Hjortnaes J, Algra A, Olijhoek J, Huisman M, Jacobs J, van der Graaf Y, Visseren F: Serum uric acid levels and risk for vascular diseases in patients with metabolic syndrome. J Rheumatol 2007;34:1882-1887.

14 López-Suárez A, Elvira-González J, Bascuñana-Quirell A, Rosal-Obrador J, MichánDoña A, Escribano-Serrano J, Benítez-Rodríguez E: Grupo para el Estudio del Riesgo Vascular Alcalá: Serum urate levels and urinary uric acid excretion in subjects with metabolic syndrome. Med Clin (Barc) 2006;126 321-324.

15 Puig JG, Martinez MA, Mora M, Fraile JM, Montoya F, Torres RJ: Serum urate, metabolic syndrome, and cardiovascular risk factors. A population-based study. Nucleosides Nucleotides Nucleic Acids 2008;27:620-623.

16 Levey AS, Bosch JP, Lewis JB, Greene T, Rog ers N, Roth D: A more accurate method to estimate glomerular filtration rate from serum creatinine: a new prediction equation. Modification of Diet in Renal Disease Study Group. Ann Intern Med 1999;130:461-470.

-17 Smith MC, Moran P, Ward MK, Davison JM: Assessment of glomerular filtration rate during pregnancy using the MDRD formula. BJOG 2008;115:109-112.

18 Bainbridge SA, von Versen-Hoynck F, Roberts JM: Uric acid inhibits placental system an amino acid uptake. Placenta 2009;30: 195-200.
19 Rott KT, Agudelo CA: Gout. JAMA 2003; 289:2857-2860

-20 Johnson RJ, Kang DH, Feig D, Kivlighn S, Kanellis J, Watanabe S, Tuttle KR, Rodriguez-Iturbe B, Herrera-Acosta J, Mazzali M: Is there a pathogenetic role for uric acid in hypertension and cardiovascular and renal disease? Hypertension 2003;41:1183-1190.

-21 Puig JG, Martinez MA: Hyperuricemia, gout and the metabolic syndrome. Curr Opin Rheumatol 2008;20:187-191.

$>22$ Chang FM, Chow SN, Huang HC, Hsieh FJ, Chen HY, Lee TY, Ouyang PC, Chen YP: The placental transfer and concentration difference in maternal and neonatal serum uric acid at parturition: comparison of normal pregnancies and gestosis. Biol Res Pregnancy Perinatol 1987;8:35-39.

23 DI, Nakagawa T, Karumanchi SA, Oliver WJ, Kang DH, Finch J, Johnson RJ: Hypothesis: Uric acid, nephron number, and the pathogenesis of essential hypertension. Kidney Int 2004;66:281-287.

$\checkmark 24$ Weisz B, Cohen O, Homko CJ, Schiff E, Sivan E: Elevated serum uric acid levels in gestational hypertension are correlated with insulin resistance. Am J Perinatol 2005;22:139144. 